\title{
EUIDENCE FOR EXERCISE TRAINING IN AUTONOMIC FUNCTION MODULATION IN PATIENTS WITH CHRONIC OBSTRUCTIVE PULMONARY DISEASE (COPD): A SYSTEMATIC REVIEW
}

\section{Mbril Mohommed1,2, Eric Derom³, Jessice Von Oosterwijck ${ }^{1,4}$, Hellen da Silva1, Patrick Caldersi \\  \\ UNIVERSITEIT GENT

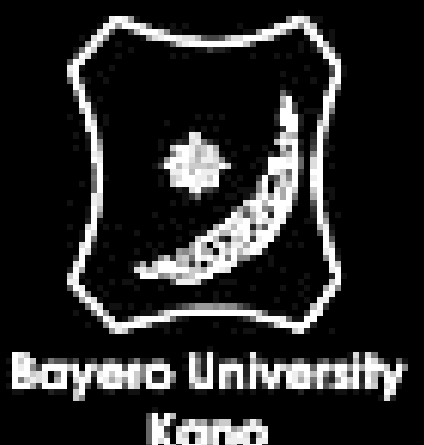 \\ $\operatorname{con}$ \\ 1 Dept. of Rehabilitation Sciences and Physical Therapy, Ghent University, Belgium. \\ 2 Dept. of Physiotherapy Bayero University, Kano, Nigeria. \\ 3 Depto of Respiratory Medicine, Ghent University, Belgium. \\ 4 Research Foundation -Flanders (FWO) Brussels, Belgium.}

\section{Aims}

To summarize the current evidence concerning the effect of exercise training on autonomic function in patients with Chronic Obstructive Pulmonary Disease (COPD).

Method's

Following the Preferred Reporting Items for Systematic reviews and Meta Analysis (PRISMA) guidelines, a systematic search was performed in three electronic databases using a combination of predefined keywords regarding COPD/exercise training and rehabilitation/outcomes of autonomic function.

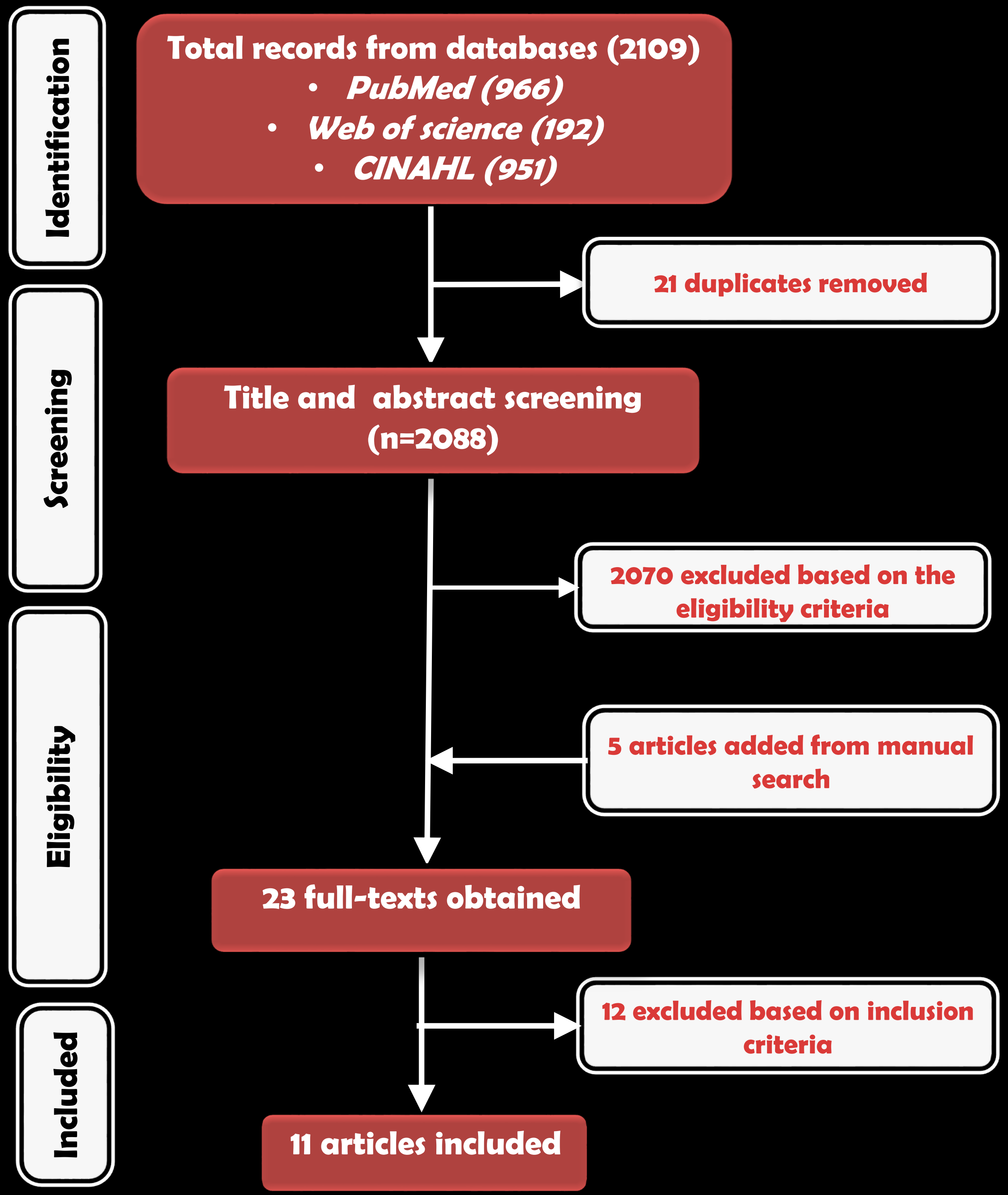

Full text reports from research studies assessing any measure of AF before and after exercise training in patients with COPD were included.

The included articles were reviewed on methodological quality, and evidence synthesis was done using the Grading of Recommendations Assessment Development, and Evaluation (GRADE) criteria.

\section{Acknowledermentse}

Jibril Mohammed is on a PhD fellowship funded by the Tertiary Education Trust Fund, Nigeria. Jessica Van Oosterwijck is a postdoctoral research fellow funded by the Research Foundation - Flanders (FWO), Belgium.

\section{Results}

- A total of 406 patients with COPD studied (279 $\hat{O}, \%$ FEV, predicted: $32 \pm 11$ to $50 \pm 19$ )

- Types of training studied:

- continuous exercise training

- vigorous intensity (60-80\% $\mathrm{VO}_{2}$ max) in majority of the studies

- Mean session duration: 30-40 min

- Number of sessions/weeks min 3x/week

- Frequent methodological issues:

- low sample sizes

- substantial drop out

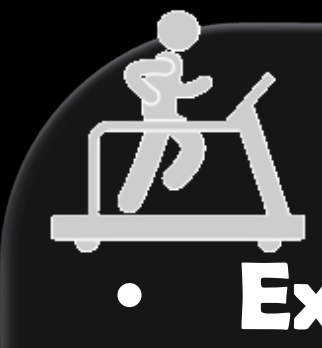
GRADE evidence synthesis

- Exercise training improves heart ráte variability (time domain) and heart rate recovery measure (high level of evidence-A).

- Only limited evidence (Level D) supported exercise training in enhancing the baroreceptor sensitivity.

- Also, exercise training appears to have no significant influence on the frequency domain parameters of heart rate variability.
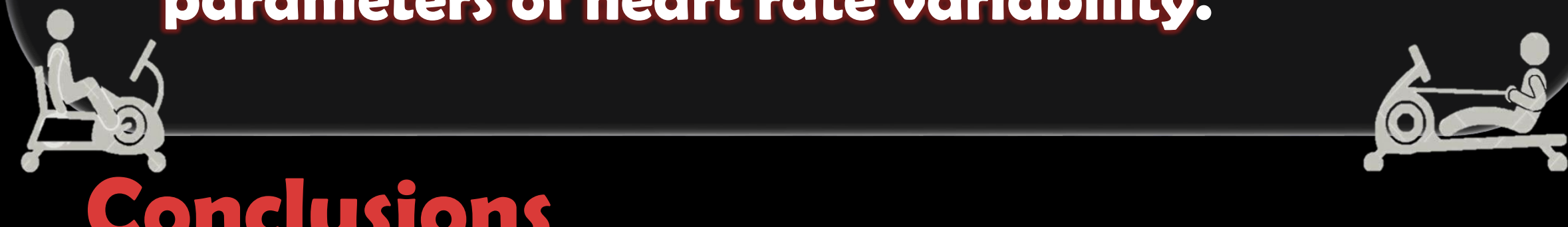

\section{concusions}

Exercise training only demonstrated limited beneficial effects for autonomic function modulation for patients with COPD. Nevertheless, future studies testing other exercise training modes with high methodological quality and large sample sizes are still necessary.

References

1. Mohammed et al . Evidence for Autonomic Function and Its Influencing Factors in Subjects With COPD: A Systematic Review. Respir Care 2015, 60:1841-1851

2. Borghi-Silva et al .Potential effect of 6 us 12-weeks of physical training on cardiac autonomic function and exercise capacity in COPD. Eur J Phys Rehabil Med2015, 51:211-21. 